\title{
Association possible entre un infarctus du myocarde sans obstruction coronarienne et l'infection par le SRAS-CoV-2
}

\author{
Shubham Agarwal MD, Haitham Al Hashimi MRCP, Sanjeev Kumar Agarwal DM, Usama Albastaki MD
}

Citation : CMAJ 2020 November 30;192:E1633-6. doi : 10.1503/cmaj.202106-f

Voir la version anglaise de l'article ici : www.cmaj.ca/lookup/doi/10.1503/cmaj.202106

$\mathbf{U}$

n homme de 26 ans est venu consulter au service des urgences en raison d'une dyspnée progressive à l'effort présente depuis 1 mois. Il n'avait pas présenté de fièvre, de toux, de douleur thoracique, de diarrhée ou de symptômes grippaux. Ses antécédents médicaux étaient sans particularité, et il était nonfumeur. À l'examen, sont pouls était de 117 battements/min, sa pression artérielle, de $135 / 100 \mathrm{~mm} \mathrm{Hg}$, et sa saturation en oxygène, de $98 \%$ à l'air ambiant. Des crépitants étaient audibles à la base des deux poumons.

Un électrocardiogramme a montré la présence d'ondes $Q$ aux dérivations $\mathrm{I}$, $\mathrm{aVL}, \mathrm{V} 5$ et $\mathrm{V} 6$, et la perte d'ondes $\mathrm{R}$ aux dérivations précordiales (figure $1 \mathrm{~A})$. Une radiographie thoracique a quant à elle révélé une cardiomégalie (figure 1B). L'analyse sanguine a donné les résultats suivants: numération leucocytaire de $12,8 \times 10^{3} / \mu \mathrm{L}$ (plage normale $3,6-11 \times 10^{3} / \mu \mathrm{L}$ ) avec $17 \%$ de lymphocytes et $0 \%$ d'éosinophiles; taux d'alanine aminotransférase de $682 \mathrm{U} / \mathrm{L}$ (plage normale $0-41 \mathrm{U} / \mathrm{L}$ ); paramètres de coagulation légèrement élevés; glycémie aléatoire de $163 \mathrm{mg} / \mathrm{dL}$ (plage normale $80-140 \mathrm{mg} / \mathrm{dL}$ ); hémoglobine glyquée $\left(\mathrm{HbA}_{1 \mathrm{c}}\right)$ de $6,7 \%$ (valeur normale $<5,7 \%$ ); taux de troponine T de $1326 \mathrm{ng} / \mathrm{L}$ (valeur

\section{POINTS CLÉS}

- Des complications cardiovasculaires, dont une nécrose myocardique importante, peuvent survenir après une infection asymptomatique ou bénigne par le coronavirus du syndrome respiratoire aigu sévère 2 (SRAS-CoV-2).

- L'évaluation des lésions myocardiques associées à l'infection par le SRAS-CoV-2 peut révéler des signes d'infarctus transmural (lésions ischémiques) et sous-endocardique (myocardite à éosinophiles) et une atteinte des deux ventricules.

normale $<14 \mathrm{ng} / \mathrm{L}$ ); taux de propeptide $\mathrm{N}$-terminal du peptide natriurétique de type B (NTproBNP) de $5166 \mathrm{pg} / \mathrm{mL}$ (valeur normale $<125 \mathrm{pg} / \mathrm{mL}$ ); et taux de D-dimères de $2,63 \mu \mathrm{g} / \mathrm{mL}$ (valeur normale $<0,5 \mu \mathrm{g} / \mathrm{mL}$ ). La numération plaquettaire, la fonction rénale et le taux de protéine $\mathrm{C}$-réactive et de ferritine du patient étaient normaux.

L'échocardiographie a mis en évidence une dilatation très importante du ventricule et de l'atrium gauches (VG et AG, respectivement), une fraction d'éjection du VG entre $15 \%$ et $20 \%$
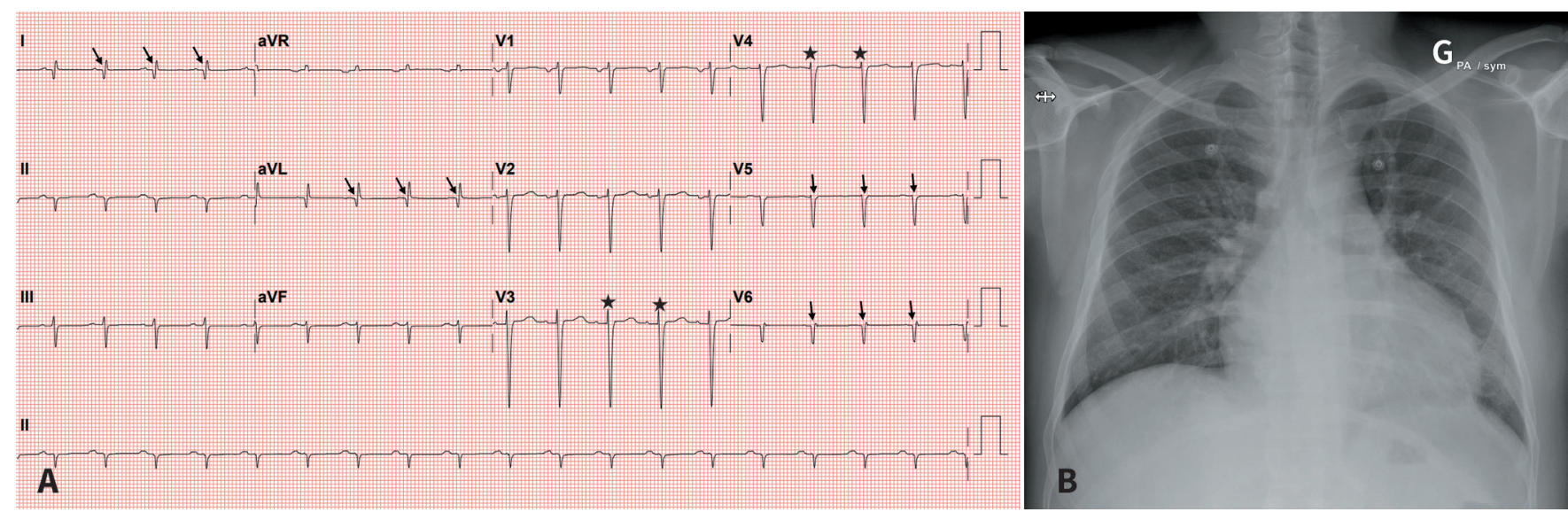

Figure 1 : A) Électrocardiogramme chez un homme de 26 ans montrant la présence d'ondes $\mathrm{Q}$ aux dérivations I, aVL, V5 et V6 (flèches) et la perte d'ondes $\mathrm{R}$ aux dérivations précordiales (astérisques). B) Radiographie thoracique révélant une cardiomégalie. 

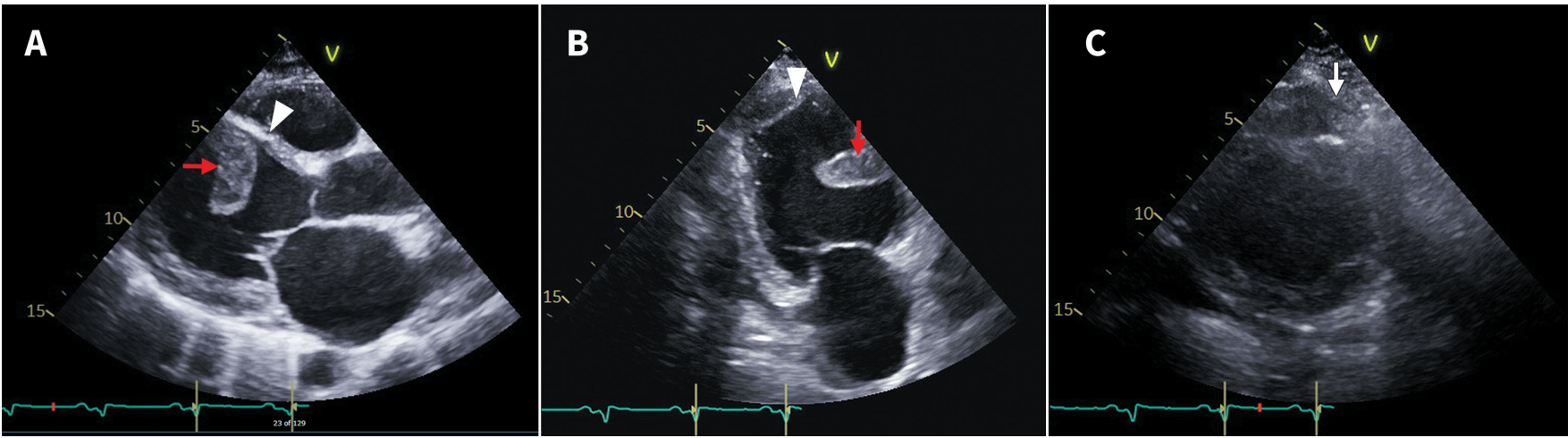

Figure 2 : Échocardiogramme à l'admission. A) Thrombus septal (flèche), septum aminci (pointe de la flèche) et ventricule gauche dilaté. B) Thrombus septal (flèche) et thrombus apical (pointe de la flèche) dans le VG. C) Thrombus apical dans le VD (flèche).
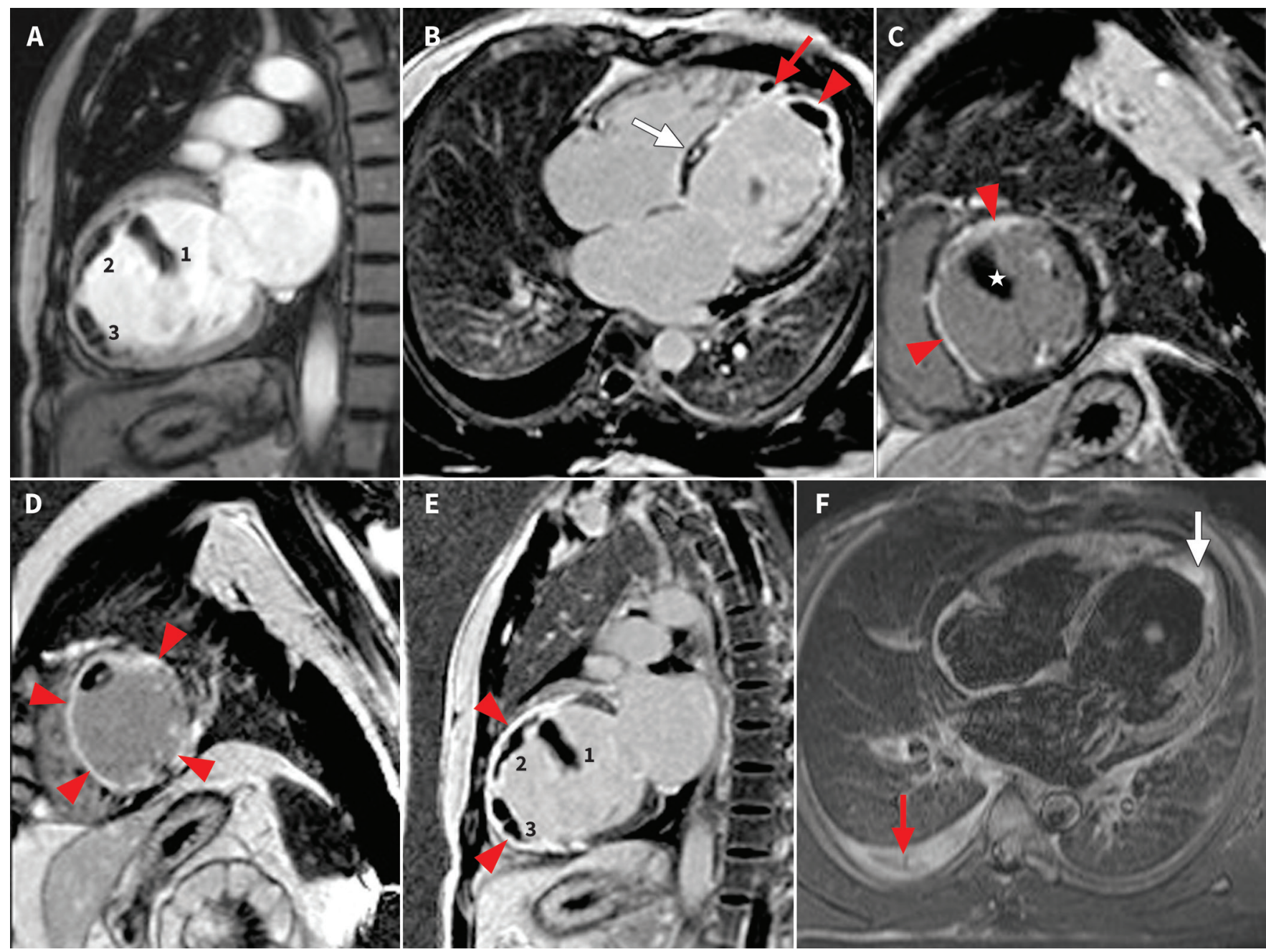

Figure 3 : Image de résonance magnétique (IRM) cardiaque (réalisée sur un système $1.5 \mathrm{~T}$ [Siemens Aera]). Dans toutes les images, les pointes des flèches rouges montrent une augmentation du contraste au niveau myocardique. A-E) Images T1 en séquence d'inversion-récupération. A) Image avec contraste au gadolinium (axe vertical longitudinal) prise dans les premiers temps et révélant 3 thrombus (numérotés) dans le VG. B) Vue des 4 cavités montrant 1) un thrombus apical dans le VG; et 2) un thrombus apical de petite taille dans le VD (flèche rouge) avec augmentation transmurale du contraste à l'apex du VG et augmentation sous-endocardique du contraste au septum. Une augmentation mi-myocardique subtile et linéaire du contraste est également observable au septum antérieur (flèche blanche). C) Vue petit axe mi-ventriculaire montrant une augmentation sous-endocardique du contraste aux parois antérieure et septale, ainsi qu'un thrombus de grande taille dans le VG (astérisque). D) Vue petit axe apical montrant une augmentation myocardique du contraste à tous les segments et un thrombus intraluminal. E) Vue de 2 cavités montrant 3 thrombus intraluminaux (numérotés 1,2 et 3) et une augmentation transmurale du contraste au septum et au myocarde inférieur. F) Image T2 en séquence d'inversion-récupération à $\tau$ court montrant un œdème endomyocardique apical (flèche blanche) et un épanchement pleural du côté droit (flèche rouge). 
et une régurgitation mitrale évaluée à $2 / 4$. On pouvait également observer une akinésie et un amincissement des segments antéroseptal et inféroseptal au niveau basal, des segments antérieur, antéroseptal et inféroseptal au niveau médian et de tous les segments au niveau apical du VG. Le patient présentait une dilatation du ventricule droit (VD), ainsi qu'une fonction systolique altérée et une régurgitation tricuspidienne modérée. On voyait aussi un thrombus mobile de grande taille sur la partie médiane de la paroi antéroseptale (figure $2 \mathrm{~A}$ ), un thrombus sessile à l'apex du VG (figure 2B) et un autre thrombus à l'apex du VD (figure 2C), de même qu'un léger épanchement péricardique.

Nous avons posé un diagnostic d'insuffisance cardiaque aiguë et hospitalisé le patient. Les diagnostics différentiels comprenaient un syndrome coronarien aigu, une myocardite, une myocardiopathie dilatée et une thromboembolie pulmonaire. Les artères coronaires avaient une apparence normale à la coronarographie, et la possibilité d'embolie pulmonaire a été éliminée à l'angiographie par tomodensitométrie. Les symptômes récents du patient et son taux très élevé de troponine ont permis d'écarter le diagnostic de cardiomyopathie dilatée.

L'imagerie par résonance magnétique cardiaque (IRM cardiaque) a montré une dilatation du VG, qui avait une fraction d'éjection de $20 \%$. La cartographie T1 et l'imagerie avec injection de gadolinium réalisées dans les premiers temps ont révélé la présence de 3 thrombus dans le VG (figure $3 \mathrm{~A}$ ); l'imagerie réalisée plus tard a mis en évidence une légère augmentation linéaire du contraste dans le segment antérobasal, au niveau miventriculaire (figure 3B). Fait notable, on a également constaté une augmentation endomyocardique du contraste, allant de la base du septum au niveau mi-ventriculaire des segments antérieur et septal (figure 3C); l'augmentation était transmurale et circonférentielle au niveau apical (figure 3D). De plus, il était possible d'observer une fibrose des segments mis en évidence par l'agent de contraste, un amincissement important du septum et une dysfonction connexe de la paroi environnante.

Comme le coronavirus du syndrome respiratoire aigu sévère 2 (SRAS-CoV-2) pouvait être à l'origine de l'insuffisance cardiaque, nous avons réalisé un test de dépistage par amplification en chaîne par polymérase couplée à une transcription inverse (RTPCR) en temps réel (Cepheid Inc., Sunnyvale, États-Unis) sur un échantillon nasopharyngé à l'admission et 72 heures plus tard; dans les 2 cas, les résultats étaient négatifs. Cependant, un test de détection d'immunoglobulines G (IgG) dirigées contre la maladie à coronavirus 2019 (COVID-19) (système ARCHITECT pour le dosage des IgG anti-SRAS-CoV-2, Abbott, États-Unis) a donné un résultat positif. Nous avons alors proposé la réalisation de tests hormonaux en raison des changements endocrinologiques pouvant survenir durant la phase de convalescence de la COVID-19. Le patient avait un taux de cortisol matinal de $30 \mathrm{nmol} / \mathrm{L}$ (plage normale 133-537 nmol) et un taux de corticotrophine inférieur à $7,1 \mathrm{pg} / \mathrm{mL}$ (plage normale $10-46 \mathrm{pg} / \mathrm{mL}$ ). Sa fonction thyroïdienne était normale.

Nous avons traité le patient avec du furosémide, du ramipril, du bisoprolol, de la spironolactone et de la warfarine, ainsi qu'avec une dose thérapeutique d'énoxaparine pour son insuffisance cardiaque. Aucun traitement antiviral n'a été administré pour contrer la COVID-19. Les symptômes se sont progressivement résorbés sur une période de 2 semaines, et le taux de troponine $T$ a diminué après quelques jours, ce qui concorde avec une lésion myocardique aiguë. L'échocardiogramme réalisé avant le congé ( 12 jours après la consultation initiale) n'a pas montré de thrombus résiduels dans les ventricules (annexe 1, accessible en anglais au www.cmaj.ca/lookup/doi/10.1503/ cmaj.202106/tab-related-content).

\section{Discussion}

Il n'est pas rare qu'une atteinte cardiovasculaire survienne durant la phase aiguë de l'infection par le SRAS-CoV-2. Selon une étude américaine menée sur 2736 patients hospitalisés en raison de la COVID-19, le tiers des patients présentaient une augmentation du taux de troponine, un indice de lésion myocardique; ces patients avaient un risque accru de décès, comparativement aux patients ayant un taux de troponine normal ${ }^{1}$. Des anomalies à l'échographie cardiaque ont été signalées chez environ la moitié des patients atteints de la COVID-192, mais on en sait peu sur les lésions cardiaques persistantes et les séquelles chroniques de la maladie après la résolution de la phase aiguë.

Comme l'ont montré les résultats négatifs de l'analyse des échantillons nasopharyngés et les résultats positifs des tests de détection d'IgG, le patient a probablement été infecté par le SRAS-CoV-2. Il est possible que sa dyspnée progressive à l'effort et son insuffisance cardiaque aient été liées à une infection aiguë par le virus. Le test de détection des anticorps utilisé aurait une spécificité de 99,6\% (intervalle de confiance [IC] à 95\% 99,199,9) et une sensibilité de 93,9\% (IC à 95\% 86,3-98,0) pour les échantillons prélevés plus de 14 jours après l'apparition des symptômes ${ }^{3,4}$. Nous avons pris en considération 4 troubles qui auraient pu être associés à l'insuffisance cardiaque aiguë du patient : un syndrome coronarien aigu, une myocardite, une myocardiopathie dilatée et un infarctus du myocarde sans obstruction coronarienne. De ces 4 troubles, l'infarctus est le plus probable.

L'absence d'angine, de sus-décalage du segment ST et de changement de l'onde $T$ attribuable à une ischémie aux électrocardiogrammes ainsi que l'obtention de résultats normaux à la coronarographie ont rendu peu probables les diagnostics de syndrome coronarien aigu, de spasme coronaire, de dissection et de thromboembolie. Par ailleurs, les résultats d'imagerie du patient, considérés ensemble ou séparément, ne concordaient pas avec une myocardite aiguë ni avec une myocardiopathie dilatée non ischémique. À l'IRM cardiaque, le patient présentait des signes subtils de myocardite aiguë, mais pas d'œdème myocardique associé, ce qui ne concordait pas avec l'importante dysfonction ventriculaire gauche et l'insuffisance cardiaque aiguë. L'augmentation tardive du contraste avec le gadolinium, la fibrose myocardique et l'amincissement important du septum interventriculaire à l'IRM cardiaque laissaient soupçonner un infarctus étendu touchant les régions irriguées par l'artère coronaire antérieure descendante gauche et l'artère coronaire droite, ce qui n'était pas compatible avec une myocardiopathie dilatée non ischémique. Nous avons donc envisagé la possibilité d'un 
infarctus du myocarde sans obstruction coronarienne en nous basant sur l'âge du patient ainsi que sur l'absence d'obstruction coronarienne combinée aux signes d'infarctus transmural ${ }^{5}$.

Les taux de troponine T et de NT-proBNP du patient étaient très élevés sans que les taux de marqueurs inflammatoires soient hauts, ce qui est cohérent si l'hospitalisation est survenue dans la phase de convalescence de la COVID-196. D'autres facteurs appuyaient indirectement cette association, soit l'apparition récente d'un diabète sucré et une suppression de l'axe hypothalamo-hypophyso-surrénalien, 2 troubles signalés chez le patient après l'infection par le SRAS-CoV- $2^{7}$. Pourtant, le patient n'a présenté aucun symptôme respiratoire classique d'infection avant et pendant son hospitalisation.

Une coronaropathie sans obstruction accompagnée d'un infarctus du myocarde a été signalée chez des patients hospitalisés pour la COVID-198,9, et une augmentation du contraste dû à l'ischémie à l'IRM cardiaque a été observée chez $12 \%$ des patients guéris de la COVID-1910. Malgré tout, il demeure difficile d'établir avec certitude un lien causal entre le SRAS-CoV-2 et un infarctus du myocarde sans obstruction coronarienne.

Les mécanismes qui sous-tendent les lésions cardiaques sont méconnus en contexte de COVID-19. Il est possible que plusieurs thrombus microvasculaires soient à l'origine des signes prédominants d'infarctus, mais ils ne seraient pas répartis uniformément dans le cœur, comme dans la pathologie cardiaque signalée chez une patiente atteinte de la COVID-19 ${ }^{11}$. Des thrombus microvasculaires pourraient expliquer la dysfonction biventriculaire et l'infarctus transmural apparent sans obstruction coronarienne chez notre patient, thrombus qui pourraient être survenus en même temps qu'une infection virale directe du cœur.

L'infection par le SRAS-CoV-2 a été associée à plusieurs symptômes cardiovasculaires. Notre étude de cas indique qu'il est possible qu'elle s'accompagne d'importantes lésions myocardiques. Le suivi minutieux des patients qui se remettent de la COVID-19 pourrait corroborer cette association et faciliter la prise en charge rapide.

\section{Références}

1. Lala A, Johnson KW, Januzzi JL, et al. Prevalence and impact of myocardial Injury in patients hospitalized with COVID-19 infection. J Am Coll Cardiol 2020; 76:533-46.

2. Dweck MR, Bularga A, Hahn RT, et al. Global evaluation of echocardiography in patients with COVID-19. Eur Heart J Cardiovasc Imaging 2020;21:949-58.

3. Bryan A, Pepper G, Wener MH, et al. Performance characteristics of the Abbott Architect SARS-CoV-2 IgG assay and seroprevalence in Boise, Idaho. J Clin Microbiol 2020;58:e00941-20.

4. Duggan J. Evaluation of the Abbott SARS-CoV-2 IgG for the detection of antiSARS-CoV-2 antibodies. London (UK): Public Health England; 2020 June 8. assets .publishing.service.gov.uk/government/uploads/system/uploads/attachment data/file/890566/Evaluation_of_Abbott_SARS_CoV_2_IgG_PHE.pdf (consulté le $1^{\text {er }}$ oct. 2020).

5. Agewall S, Beltrame JF, Reynolds HR, et al. on behalf of the WG on cardiovascular pharmacotherapy. ESC working group position paper on myocardial infarction with non-obstructive coronary arteries. Eur Heart J 2017;38:143-53.

6. Ma KL, Liu ZH, Cao CF, et al. COVID-19 myocarditis and severity factors: an adult cohort study. medRxiv 2020 Mar. 23. doi: 10.1101/2020.03.19.20034124v1 (consulté le 29 oct. 2020).

7. Agarwal S, Agarwal SK. Endocrine changes in SARS-CoV-2 patients and lessons from SARS-CoV. Postgrad Med J 2020;96:412-6.

8. Bangalore S, Sharma A, Slotwiner A, et al. ST-segment elevation in patients with COVID-19: a case series. N Engl J Med 2020;382:2478-80.

9. Calvillo-Argüelles O, Ross HJ. Cardiac considerations in patients with COVID-19. CMAJ 2020;192:E630.

10. Puntmann VO, Carerj ML, Wieters I, et al. Outcomes of cardiovascular magnetic resonance Imaging in patients recently recovered from coronavirus disease 2019 (COVID-19). JAMA Cardiol 2020 July 27 [Cyberpublication avant impression]. doi: 10.1001/jamacardio.2020.3557.

11. Guagliumi G, Sonzogni A, Pescetelli I, et al. Microthrombi and ST-segment elevation myocardial infarction in COVID-19. Circulation 2020;142:804-9.

\section{Intérêts concurrents : Aucun déclaré.}

Cet article a été révisé par des pairs.

Les auteurs ont obtenu le consentement du patient.

Affiliations : Faculté de médecine de Chicago, Université de médecine et de science Rosalind Franklin (Shubham Agarwal), Nord de Chicago (Illinois); Service de cardiologie (Haitham Al Hashimi, Sanjeev Kumar Agarwal) et Service de radiologie (Usama Albastaki), Hôpital Rashid, Dubai (Émirats arabes unis).
Collaborateurs : Shubham Agarwal et Sanjeev Kumar Agarwal ont contribué à l'élaboration et à la conception de l'étude, recueilli, analysé et interprété les données, et rédigé la première version du manuscrit. Tous les auteurs ont participé à la révision et à la correction du texte, ont approuvé la version finale aux fins de publication et assument l'entière responsabilité de tous les aspects du travail.

Propriété intellectuelle du contenu : Il s'agit d'un article en libre accès distribué conformé- ment aux modalités de la licence Creative Commons Attribution (CC BY-NC-ND 4.0), qui permet l'utilisation, la diffusion et la reproduction dans tout médium à la condition que la publication originale soit adéquatement citée, que l'utilisation se fasse à des fins non commerciales (c.-à-d., recherche ou éducation) et qu'aucune modification ni adaptation n'y soit apportée. Voir : https://creativecommons.org/ licenses/by-nc-nd/4.0/

Correspondance : Sanjeev Kumar Agarwal, skacardio@yahoo.com 